\title{
Clinical Guideline Highlights for the Hospitalist: Diagnosis and Management of Clostridium difficile in Adults
}

\author{
Andrew S Parsons, MD, MPH ${ }^{1, *}$, Alfred Burger, MD, MS², Amit K Pahwa, MD³
}

'University of Virginia School of Medicine, Charlottesville, Virginia; ${ }^{2}$ Mount Sinai Beth Israel, Icahn School of Medicine at Mount Sinai, New York, New York; ${ }^{3}$ Johns Hopkins University School of Medicine, Baltimore, Maryland.

GUIDELINE TITLE: 2018 Infectious Disease Society of America (IDSA)/Society for Healthcare Epidemiology of America (SHEA) Clostridium difficile infection (CDI) clinical practice guideline

RELEASE DATE: February 15, 2018

PRIOR VERSION: 2010 Infectious Disease Society of America (IDSA)/Society for Healthcare Epidemiology of America (SHEA) Clostridium difficile infection (CDI) clinical practice guideline

DEVELOPER: A panel of 14 multidisciplinary experts

FUNDING SOURCE: IDSA and SHEA

TARGET POPULATION: Adult and pediatric patients at risk for or diagnosed with Clostridium difficile infection based on a literature review with a defined search period of 2009-2016. This review will focus on adult patients.

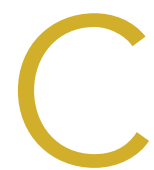

lostridium difficile, now referred to as Clostridioides difficile (C. difficile), is the most commonly identified cause of healthcare-associated infection among adults in the United States. ${ }^{1}$ Because C. difficile infection results in significant mortality and inpatient costs, its persistence threatens to undermine patient safety and the value of healthcare delivery. ${ }^{1}$ A standardized, evidence-based approach to diagnosis and management is crucial. However, inconsistencies remain with regard to the appropriate threshold for testing, the type of diagnostic tests used, and treatment. Knowledge of these areas has progressed since the publication of the previous $C$. difficile guidelines in 2010. These guidelines contain 53 recommendations across 35 sections based on a systematic weighting of the strength of recommendation and quality of evidence using the Grading of Recommendations Assessment, Development, and Evaluation system. Herein, we have chosen to highlight five of these recommendations most relevant to hospitalists.

\section{KEY RECOMMENDATIONS FOR THE HOSPITALIST}

Recommendation 1. Patients with unexplained and new-onset $\geq 3$ unformed stools within 24 hours are the preferred target population for testing for $C$. difficile infection (weak recommendation, very low quality of evidence). Do not perform

*Corresponding Author: Andrew S. Parsons, MD, MPH; E-mail: asp5c@virginia. edu; Telephone: 434-0243-0763; Twitter: @andrewparsonsMD

Published online first September 18, 2019.

Received: May 20, 2019; Revised: August 6, 2019; Accepted: August 6, 2019

(C) 2020 Society of Hospital Medicine DOI 10.12788/jhm.3300 repeat testing (within seven days) during the same episode of diarrhea and do not test stool from asymptomatic patients (strong recommendation, moderate quality of evidence).

In the recent past, healthcare facilities employed $C$. difficile tests with limited sensitivity, leading to frequent and repeat testing of hospitalized patients. Excess testing puts patients at risk for false positive results and unnecessary or prolonged treatment courses. Proper testing requires consideration of pretest probability, including analysis of the alternative causes of diarrhea. Duration of hospitalization and antibiotic exposure are the most significant modifiable risk factors for $C$. difficile infection in adult inpatients. ${ }^{2}$ Laxative use within the previous 48 hours, enteral tube feeding, and underlying medical conditions, such as inflammatory bowel disease (IBD), are common causes of improper testing. ${ }^{3}$ This decision may be difficult, as some underlying causes of diarrhea, such as IBD and enteral tube feeding, also increase the risk of $\mathrm{C}$. difficile infection. ${ }^{3}$ Laboratories can help by rejecting specimens that are not liquid or soft and employing a multistep algorithm using a combination of nucleic acid testing, antigen testing, and toxin detection to maximize sensitivity and specificity. Because recurrent $C$. difficile infection is relatively common, repeat testing is appropriate only for recurrence of symptoms following successful treatment and should focus on detection of $C$. difficile toxin because the persistence of the organism itself can occur after successful treatment. ${ }^{4}$

Recommendation 2. Either vancomycin ( $125 \mathrm{mg}$ orally four times per day for 10 days) or fidaxomicin (200 mg twice daily for 10 days) is recommended over metronidazole for an initial episode of nonsevere or severe $C$. difficile infection (strong recommendation, high quality of evidence). For fulminant $C$. difficile infection, the regimen of choice is a vancomycin dos- 
age of $500 \mathrm{mg}$ orally four times per day (per rectum every six hours if with ileus) in addition to intravenous metronidazole (strong recommendation, moderate quality of evidence).

For several decades now, metronidazole has been the primary antibiotic agent for initial treatment of nonsevere $C$. difficile infection. Two recent randomized, placebo-controlled trials, however, have found oral vancomycin to be superior to metronidazole for producing a clinical cure and resolution of diarrhea without recurrence. , $6^{6}$ Oral vancomycin remains the treatment of choice for severe $C$. difficile infection. Fidaxomicin, a recently FDA-approved antibiotic, can also be used as initial treatment in place of oral vancomycin. One study found fidaxomicin to be superior to oral vancomycin for producing a sustained clinical response, that is, resolution of diarrhea at the end of treatment without recurrence 25 days later. ${ }^{7}$ Fulminant disease, which is characterized by hypotension or shock, ileus, or megacolon, requires a higher dose of oral vancomycin (or vancomycin enema if with ileus) in addition to intravenous metronidazole.

Recommendation 3. Treat a first recurrence of $C$. difficile infection with oral vancomycin as a tapered and pulsed regimen rather than a second standard 10-day course of vancomycin or metronidazole (weak recommendation, low quality of evidence).

Despite the improved treatment response with oral vancomycin, one in four patients will experience recurrence. For a first recurrence of $C$. difficile infection after a 10-day course of oral vancomycin, an extended taper or pulsed course of vancomycin should be attempted. Various regimens have been tried and found to be effective. For a second recurrence, providers can consider addition of rifaximin following oral vancomycin. Fecal microbiota transplantation is recommended for patients with multiple recurrences of $C$. difficile infection who have failed these antibiotic treatments.

Recommendation 4. Minimize the frequency and duration of high-risk antibiotic therapy (based on local epidemiology) and the number of antibiotic agents prescribed to reduce $C$. difficile infection risk (strong recommendation, moderate quality of evidence).

Antibiotic stewardship is a necessary component of any successful effort to reduce $C$. difficile infections. Antibiotic stewardship programs, which are now commonplace in US hospitals, largely rely on educational initiatives or committee-based order review. Hospitalists should take a structured approach emphasizing the four critical questions of antibiotic prescribing: Does this infection require antibiotics? Have I ordered appropriate cultures and the correct empiric therapy? Can I stop, narrow, or switch to oral agents? Finally, what duration of therapy is needed at discharge $?^{8}$ Initial efforts should focus on the restriction of fluoroquinolones, clindamycin, and cephalosporins (except for surgical antibiotic prophylaxis) given their known risk to cause $C$. difficile infection.

Recommendation 5. Contact precautions should be maintained for at least 48 hours after diarrhea has resolved (weak recommendation, low quality of evidence).

Although $C$. difficile is undetectable in stool samples from most patients by the time diarrhea has resolved, skin and en- vironmental contaminations remain high. No studies demonstrating a benefit to further extending contact precautions beyond 48 hours after resolution of diarrhea are yet available.

\section{CRITIQUE}

\section{Methods in Preparing Guidelines}

The guideline committee consisted of an interdisciplinary team of healthcare providers with extensive experience in the diagnosis, infection control, treatment, and management of $C$. difficile. The literature search accessed five different databases (Medline, Embase, Cochrane, Health Technology Assessment, and Database of Abstracts of Reviews and Effects), relevant journals, conference proceedings, and regulatory websites published over the search period of 2009-2016.

A major strength of these guidelines is the extensive work that went into their preparation. The committee reviewed over 14,000 pieces of literature and performed a detailed analysis of each one to determine the quality of evidence in support of each recommendation.

\section{Sources of Potential Conflict of Interest or Bias}

To reduce bias, the committee's work was funded by Infectious Disease Society of America and Society for Healthcare Epidemiology of America. Some authors received funding for work outside of this guideline by companies that manufacture diagnostic assays, vancomycin, and fidaxomicin. These potential conflicts were listed at the end of the article.

\section{Generalizability of the Guideline}

Not all studies included in the guideline contain exclusively hospitalized patients, but much of the guideline content is applicable to hospitalized patients. Because $C$. difficile infection is such a widespread public health problem and these guidelines represent a significant update in knowledge since 2010, the specific recommendations highlighted in this review will impact numerous hospitalists, regardless of the practice setting.

\section{Areas in Need of Future Study}

Based on the current literature, as well as statements in the guideline, we expect future guidance around potential screening for and isolation of asymptomatic carriers, including closer guidance on stool transplantation focusing on timing and route, as further data emerge in these areas.

\section{Other Resources}

- Grading of Recommendations Assessment, Development, and Evaluation system (http://www.gradeworkinggroup.org)

- Universal Screening for C. difficile in a Tertiary Hospital: risk factors for carriage and clinical disease (https:// www.clinicalmicrobiologyandinfection.com/article/S1198743X(19)30048-5/fulltext)

- Effectiveness of Isolating Clostridium Difficile Asymptomatic Carriers on the Incidence of Infections (https://clinicaltrials. gov/ct2/show/NCT03223415)

- Effect of Detecting and Isolating Clostridium difficile Carriers at Hospital Admission on the Incidence of $\mathrm{C}$ difficile 
Infections (https://jamanetwork.com/journals/jamainternalmedicine/fullarticle/2516765)

- Clinical Trial Testing Fecal Microbiota Transplant for Recurrent Diarrheal Disease Begins (https://www.nih.gov/newsevents/news-releases/clinical-trial-testing-fecal-microbiota-transplant-recurrent-diarrheal-disease-begins)

Disclosures: The authors have nothing to disclose.

\section{References}

1. Dubberke ER, Olsen MA. Burden of Clostridium difficile on the healthcare system. Clin Infect Dis. 2012;55(2):S88-S92. https://doi.org/10.1093/cid/ cis335.

2. Loo VG, Bourgault AM, Poirier L, et al. Host and pathogen factors for Clostridium difficile infection and colonization. N Engl J Med. 2011;365(18):1693703. https://doi.org/10.1056/NEJMoa1012413.

3. O'Keefe SJ. Tube feeding, the microbiota, and Clostridium difficile infec- tion. World J Gastroenterol. 2010;16(2):139-142. https://doi.org/10.3748/wjg. v16.i2.139

4. Zacharioudakis IM, Zervou FN, Pliakos EE, Ziakas PD, Mylonakis E. Colonization with toxinogenic $C$. difficile upon hospital admission, and risk of infection: a systematic review and meta-analysis. Am J Gastroenterol. 2015;110(3):381-90; quiz 391. https://doi.org/10.1038/ajg.2015.22.

5. Johnson S, Louie TJ, Gerding DN, et al. Vancomycin, metronidazole, or tolevamer for Clostridium difficile infection: results from two multinational, randomized, controlled trials. Clin Infect Dis. 2014;59(3):345-354. https://doi. org/10.1093/cid/ciu313.

6. Zar FA, Bakkanagari SR, Moorthi KM, Davis MB. A comparison of vancomycin and metronidazole for the treatment of Clostridium difficile-associated diarrhea, stratified by disease severity. Clin Infect Dis. 2007;45(3):302-307. https:// doi.org/10.1086/519265.

7. Crook DW, Walker AS, Kean Y, et al. Fidaxomicin versus vancomycin for Clostridium difficile infection: meta-analysis of pivotal randomized controlled trials. Clin Infect Dis. 2012;55(2):S93-S103. https://doi.org/10.1093/cid/cis499.

8. Tamma, PD, Miller MA, Cosgrove SE. Rethinking how antibiotics are prescribed: incorporating the 4 moments of antibiotic decision making into clinical practice. JAMA. 2018;321(2):139-140. https://doi.org/10.1001/ jama.2018.19509. 